\title{
Amplitude death of coupled hair bundles with stochastic channel noise
}

\author{
Kyung-Joong Kim and Kang-Hun Ahn \\ Department of Physics, Chungnam National University, \\ Daejeon, 305-764, Republic of Korea
}

\begin{abstract}
Hair cells conduct auditory transduction in vertebrates. In lower vertebrates such as frogs and turtles, due to the active mechanism in hair cells, hair bundles(stereocilia) can be spontaneously oscillating or quiescent. Recently, the amplitude death phenomenon has been proposed [K.-H. Ahn, J. R. Soc. Interface, 10, 20130525 (2013)] as a mechanism for auditory transduction in frog hair-cell bundles, where sudden cessation of the oscillations arises due to the coupling between non-identical hair bundles. The gating of the ion channel is intrinsically stochastic due to the stochastic nature of the configuration change of the channel. The strength of the noise due to the channel gating can be comparable to the thermal Brownian noise of hair bundles. Thus, we perform stochastic simulations of the elastically coupled hair bundles. In spite of stray noisy fluctuations due to its stochastic dynamics, our simulation shows the transition from collective oscillation to amplitude death as inter-bundle coupling strength increases. In its stochastic dynamics, the formation of the amplitude death state of coupled hair bundles can be seen as a sudden suppression of the displacement fluctuation of the hair bundles as the coupling strength increases. The enhancement of the signalto-noise ratio through the amplitude death phenomenon is clearly seen in the stochastic dynamics. Our numerical results demonstrate that the multiple number of transduction channels per hair bundle is an important factor to the amplitude death phenomenon, because the phenomenon may disappear for a small number of transduction channels due to strong gating noise.
\end{abstract}

PACS numbers: 


\section{INTRODUCTION}

The ear amplifies incoming sound signals with high sensitivity and sharp frequency selectivity. It can detect sound stimuli over many orders of magnitude in sound amplitude. The great sensitivity of hearing originates from the active process in hair cells which perform mechano-transduction in the inner ear. Sharp frequency selectivity and active amplification arise with the aid of outer hair cell(OHC) and membrane dynamics in the mammalian cochlea, whereas non-mammals lacking in OHC such as frogs, also have acute hearing relying on hair-cell bundle dynamics[2 5]. Hair bundles can be spontaneously oscillating or quiescent depending on their physical parameters. It has been speculated that hair bundles operate on the border of these two regions, which is called Hopf-bifurcation critical point [5, 6]. Such an assumption may explain the high sensitivity and the sharp frequency selectivity of hearing.

A question one may have is whether the bifurcation phenomenon indeed exists in-vivo. In reality, it is more appropriate to assume a distribution of physical parameters of hair bundles, rather than assuming all hair bundles are equivalent as in a simple mathematical model. The dynamics of coupled hair bundles in a homogeneous configuration can be found in Ref. [7, 8]. In a recent theory, it has been demonstrated that parametric distributions of hair bundles help auditory transduction through inter-bundle coupling[1]. The phenomenon has been known as amplitude death in the nonlinear dynamics community, which has been reported in many cases of coupled oscillators [9-20]. The amplitude death phenomenon in hair cells has been introduced through continuous variable dynamics[1], where the open probabilities of ion channels are functions of the mechanical displacement of the hair bundle. Meanwhile, due to the stochastic nature of the configuration change of the ion channel, the ion channel gating in cilia happens in a stochastic manner with dwell time of $1 \mathrm{~ms}$ [21, 22]. This causes intrinsic noise associated with the stochastic channel gating.

Furthermore, as we will show, the noise associated with stochastic channel gating can be of comparable strength to the thermal noise force. Thus it is necessary to investigate whether the amplitude death exists in the presence of the intrinsic channel gating noise.

In this work, we show that the amplitude death phenomenon of coupled hair bundles indeed exists even when the bundle dynamics is treated in a stochastic manner. We simulate the dynamics of the coupled hair bundles by using the stochastic Markov process and find the 
features of the amplitude death phenomenon for the coupled hair bundles, where a rigorous model for bullfrog hair bundles has been used. We find that the statistical distribution of the bundles' displacement shows a transition from a bimodal to a single modal structure as we increase the inter-bundle coupling strength. The displacement distribution of hair bundles in the amplitude death state turns out to be weakly asymmetric. The velocity distribution of the hair bundles shows transition from the half-Lorentizian to Gaussian distribution. In the region of spontaneous oscillation, the hair bundle velocity distribution is asymmetric Lorentzian due to the interplay between relaxation and the active process. In the amplitude death region, velocity distribution is well described by the Gaussian distribution. It is demonstrated that the signal-to-noise ratio and the response of the coupled hair bundle to an oscillatory stimulus is enhanced as the system is located in the amplitude death region.

The relative importance of the stochastic channel gating noise is discussed by comparing it to the thermal noise force on the hair bundle displacement. We will show that the stochastic noise can be reduced by increasing the number of the ion channels per hair bundle. For the hair bundles which have a few tens of ion channels, we will show the stochastic channel gating noise is sufficiently weakened so that the amplitude death phenomenon survives both types of noise.

The paper is organised as follows. In Section II, we introduce the stochastic Markov model for the elastically coupled hair bundles. The calculation results for the stochastic hair bundle model are shown and discussed in Section III. We demonstrate the amplitude death in the stochastic dynamics in III A and the enhanced signal-to-noise ratio in III B. The thermal noise and the stochastic channel-gating noise are compared and the significance of the number of channels per hair bundle is discussed in III C. The conclusion is given in Section IV.

\section{STOCHASTIC DYNAMICS MODEL OF COUPLED HAIR BUNDLES}

Hair bundles in a bullfrog sacculus are coupled by the otolithic membrane which has a finite mass [23]. We consider a one-dimensional hair bundle array which is elastically coupled with finite mass elements attached to it in Fig. 1 (a). The equations governing the dynamics of the coupled hair bundles are

$$
m \ddot{x}_{i}=-m \gamma_{m} \dot{x}_{i}+k\left(x_{i+1}-2 x_{i}+x_{i-1}\right)+f_{\mathrm{HB}, i}
$$


where $x_{i}$ is the displacement of $i$-th hair bundle $(i=1,2,3, \cdots, N)$ and $N$ is the number of hair bundles. Here, we use finite elements of mass $m$ to describe the loading effect of the overlying membrane. $k$ is the inter-bundle coupling strength and $\gamma_{m}$ is the friction constant per mass, which was chosen to mimic the poor frequency selectivity of the overlying membrane[1].

The force exerted on the $i$-th element by the $i$-th hair bundle is

$$
f_{\mathrm{HB}, i}=-\lambda \dot{x_{i}}-k_{g s}\left(x_{i}-x_{a, i}-\frac{D}{N_{c h}} \sum_{j=1}^{N_{c h}} G_{i, j}\right)-k_{s p, i} x_{i},
$$

where the pivotal stiffness and the combined gating spring stiffness are denoted by $k_{s p}$, and $k_{g s}$, respectively. $D$ is the displacement associated with channel gating and $\lambda$ is the friction coefficient of the hair bundle[5]. To simulate the dynamics of the multiple number of transduction channels per hair bundle, we assume $N_{\mathrm{ch}}$ number of transduction channels. For simplicity, we assume that all the molecular motor positions of the $i$-th hair bundle are equal to $x_{a, i}$. Here, $G_{i, j}=1(0)$ when the $j$-th channel of $i$-th bundle is open (closed). The probability for $G_{i, j}$ to be 1 is given by the open probability of the $i$-th ion channel, $p_{o, i} . x_{a, i}$ is the position of the molecular motor in the $i$-th hair bundle, which satisfies

$$
\lambda_{a} \dot{x}_{a, i}=k_{g s}\left(x_{i}-x_{a, i}-\frac{D}{N_{c h}} \sum_{j=1}^{N_{c h}} G_{i, j}\right)-f_{\max , i}\left(1-S p_{o, i}\right),
$$

where $f_{\max , i}\left(1-S p_{o, i}\right)$ is the force exerted by the molecular motor and $\lambda_{a}$ is the velocity-force relation constant of the molecular motor [5]. $f_{\max , i}$ is the motor's maximal force and $S$ is a parameter for the strength of the calcium feedback [5]. Since the feedback force strength is a function of the calcium ion concentration in the stereocilia, we think it is more natural to assume that the feedback force is a function of the mean open probability $p_{o, i}$ rather than the number of open channels at a particular time.

In vivo, the open-closed transitions of the ion channels in cilia occurs stochastically with a dwell time of $1 \mathrm{~ms}[21]$, which is our motivation to describe our model for coupled hair bundles in the Markov process.

See Fig. 1. Let us consider a two-state channel which has the energy $E_{o}$ in the open state and $E_{c}$ in the closed state. The equilibrium transition rates of these two states, $\Gamma_{o c}$ and $\Gamma_{c o}$, are related by the Boltzmann equation $\frac{\Gamma_{o c}}{\Gamma_{c o}}=e^{-\left(E_{o}-E_{c}\right) / k_{B} T}=e^{-\Delta E / k_{B} T}\left(k_{B}\right.$ is the Boltzmann constant, and $T$ is the temperature) where $\Delta E$ is the energy difference associated with the channel gating. The evolution of a continuous-time process is given by 
the first-order differential equation

$$
\frac{d p_{o}}{d t}=\Gamma_{o c}\left(1-p_{o}\right)-\Gamma_{c o} p_{o}
$$

In the case of $\frac{d p_{o}}{d t} \cong 0$, we obtain the open probability by

$$
p_{o} \cong \frac{\Gamma_{o c}}{\Gamma_{c o}+\Gamma_{o c}}=1 /\left(1+e^{\Delta E / k_{B} T}\right)
$$

The channel gating in hair cells is caused by mechanical stimulation which is delivered by a tip link, a filament interconnecting adjacent stereocilia[24]. Since the mechanical energies stored in the gating springs are $E_{o}=\frac{1}{2} k_{g s}\left(x-x_{a}-D\right)^{2}$ and $E_{c}=\frac{1}{2} k_{g s}\left(x-x_{a}\right)^{2}$, the open probability of the $i$-th hair bundle is given by

$$
p_{o, i}=1 /\left(1+A \exp \left(-\frac{x_{i}-x_{a, i}}{\delta}\right)\right)
$$

where $A$ is a constant associated with the intrinsic free energy difference between the open and closed states and $\delta=k_{B} T N_{c h} /\left(k_{g s} D\right)$ is a typical length associated with the channel gating [5, 25, 26]. Note that this length $\delta$ is independent of the number of channel $N_{c h}$, because the combined gating spring stiffness $k_{g s}$ is proportional to $N_{c h}$.

At each time step with interval $\Delta t$, we generate random numbers $\xi_{i, j}(i=1,2, \cdots, N$ and $\left.j=1,2, \cdots, N_{c h}\right)$ which are distributed between 0 and 1 , and we compare then with the transition probabilities,

$$
\begin{aligned}
& \omega_{o c, i}=\gamma \Delta t p_{o, i} \\
& \omega_{c o, i}=\gamma \Delta t\left(1-p_{o, i}\right)
\end{aligned}
$$

where $\gamma$ is a parameter giving the channel relaxation rate. When the channel is in the open state $\left(G_{i, j}=1\right)$, it remains in the open state if $\omega_{c o, i}<\xi_{i, j}$, or changes to the closed state $\left(G_{i, j}=1 \rightarrow G_{i, j}=0\right)$ if $\omega_{c o, i}>\xi_{i, j}$. Similarly, when the channel is in the closed state, the state changes to the open $\operatorname{state}\left(G_{i, j}=0\right)$, if $\omega_{o c, i}>\xi_{i, j}\left(G_{i, j}=0 \rightarrow G_{i, j}=1\right)$.

The thermal fluctuation of the channel gate affects the bandwidth of the intrinsic stochastic noise. The fluctuation-dissipation theorem says that as the fluctuation gets larger, it accompanies a larger dissipation. Since, in our simulation, the bandwidth of the channel gating noise is given by $1 / \Delta t$, and the relaxation rate of the gating is about $\gamma$, these two values should be in the same order of magnitude to fulfill the fluctuation dissipation theorem. Thus, throughout this work, we choose $\gamma \Delta t=0.4$. As will be shown here, our stochastic 
simulation results resemble those of the continuous variable calculations [1] for a sufficiently large relaxation rate $\gamma$ or a large number of ion channels per bundle $N_{c h}$, which is likely the case in biological systems. In contrast, when the relaxation of the ion channel is not fast enough the channel gating noise becomes too strong and might destroy the amplitude death phenomenon.

\section{RESULTS AND DISCUSSION}

As discussed in Ref.[1], an inhomogeneous distribution of the physical parameters of hair bundles is likely to exist in biological systems and is critical to the emergence of amplitude death. We simulate the non-uniformity of the hair bundles by using a distribution of the bundle's pivotal stiffness $k_{s p}$ and the maximal motor force $f_{\max }$ (Fig. 2 (a)). The parameters for hair bundles are given to ensure that some of them are closed and the others are spontaneously oscillating.

\section{A. SUPPRESSION OF MECHANICAL FLUCTUATION OF COUPLED HAIR} BUNDLES

To investigate the existence of amplitude death in a stochastic nature, it proves useful to calculate the histogram for the displacement of the hair bundles as shown in Fig. 2 (b) $\sim(g)$. These histograms depict the number of events for the hair bundles to be located in the given displacement interval. The events are counted every millisecond for 2 seconds. In the absence of coupling(Fig. 2 (b)), the histogram shows a multiple-peak structure, which represents the different oscillation states or closed states for each of the hair bundles. Since we assume that the ion channels are either closed or oscillatory, the peaks are mainly on the position of the closed state rather than on the open state. At weak couplings(Fig. 2 (c),(d),(e)), the histograms show two main peaks. As the coupling strength increases, the distance between these two main peaks becomes reduced. Above a certain coupling strength, the histogram shows only one peak corresponding to the closed state(Fig. 2 (f),(g)). The tail of the peak in the histogram rapidly disappears as the strength increases further(Fig. $2(\mathrm{~g}))$. This corresponds to the amplitude death phenomenon of the coupled hair bundles which has been shown in the continuum model for hair bundles[1]. 
The transition to the amplitude death region can also be seen through the velocity distribution. We find that it has a half-Lorentzian distribution in the collectively oscillating states and shows a transition to Gaussian distribution(Fig. 3). The underlying mechanism for the half-Lorentzian distribution arises from the bundles opening slowly and closing quickly due to the relaxation process and active oscillation(inset of Fig. 3). In the amplitude death region, random channel gating noise causes the Gaussian distribution of the velocity.

In Fig. 4, we show the variances for the displacements of the coupled hair bundles which are calculated from the stochastic and continuum model. The variance $\sigma_{X}^{2}$ is the average of the variance of the displacement of each single hair bundle

$$
\begin{aligned}
\sigma_{X}^{2} & =\frac{1}{N} \sum_{i} \sigma_{X, i}^{2} \\
\sigma_{X, i}^{2} & \left.=<\left(x_{i}-<x_{i}>_{t}\right)^{2}\right\rangle_{t},
\end{aligned}
$$

where $\left\langle>_{t}\right.$ means the average over time. The error bars in Fig. 4 are estimated from 20 trials of the simulations. The $\sigma_{X}$ value shows a good agreement with the values which were obtained from a continuum model when the coupling is not sufficiently strong enough to cause amplitude death. When the coupling strength is sufficiently strong, the fluctuation of coupled hair bundles in the stochastic model also shows a dramatic reduction as in the continuum model calculation of Ref.[1]. Thus, even in the presence of the channel gating fluctuations, the strong reduction of the mechanical fluctuation appears as the fingerprint of the amplitude death phenomenon, which was originally considered in noise-free dynamics $[9$ 20].

While the transition to the amplitude death region is clearly seen in the distribution of the displacement and velocity, the normalized correlation between the hair bundles does not have any significant difference in these two regions. We define the correlation between hair bundle displacements by $C=\frac{2}{N} \sum_{i \neq j}\left\{<x_{i} x_{j}>_{t}-<x_{i}>_{t}<x_{j}>_{t}\right\}$. We find that this correlation function is also suppressed in the amplitude death region (see Supplemental Material Fig. 9 (a)). We define the normalized correlation function $C_{N}$ for the displacement of the coupled hair bundles which is given by $C_{N} \equiv\left[1+2\left(\frac{\left.\left.\sum_{i \neq j}\left(\left\langle x_{i} x_{j}\right\rangle_{t}-<x_{i}\right\rangle_{t}<x_{j}\right\rangle_{t}\right)}{\sum_{i}\left(\left\langle x_{i}^{2}>_{t}-\left\langle x_{i}\right\rangle_{t}^{2}\right)\right.}\right)\right] / N$. Then, $C_{N}$ is 0.1 at zero coupling strength but it goes up to 0.8 as $k$ increases, and retains the value regardless of amplitude death (see Supplemental Material Fig. 9 (b)). This means that movements of the hair bundles are rather coherent and they do not lose this coherence in the amplitude death region. In other words, the amplitudes of oscillations are quenched 
but their cross-correlation is maintained in the amplitude death region.

\section{B. ENHANCEMENT OF SIGNAL-TO-NOISE RATIO BY AMPLITUDE DEATH}

Now we show how the hair bundles in the amplitude death state respond to oscillatory stimuli. We investigate the time evolution of the average displacement (Fig. 5 (a)) of the coupled hair bundles in our stochastic model. We applied a periodic stimulus $F_{\text {ext }}=2 \mathrm{pN}$ $\times \sin (2 \pi \times 10 \mathrm{~Hz} \times t)$ only when 2 sec $<t<4$ sec. In the absence of any stimulus, we can see the coupled hair bundles move collectively for the coupling $k=2 \mathrm{pN} / \mathrm{nm}$. Then the collective oscillation is destroyed above a certain coupling $\operatorname{strength}(k=4.3 \mathrm{pN} / \mathrm{nm})$ where the critical coupling strength for the transition to amplitude death is not universal but depends on the hair bundles' parameter distribution (not shown). Fig. 5 (b) shows the time evolution of the ratio of open to total channels, which is defined by

$$
\begin{aligned}
\tilde{G}_{o} & =\frac{1}{N} \sum_{i=1}^{N} G_{i}, \\
G_{i} & =\frac{1}{N_{c h}} \sum_{j=1}^{N_{c h}} G_{i, j} .
\end{aligned}
$$

This shows a similar pattern with the averaged open probability $\tilde{P}_{o}=\frac{1}{N} \sum_{i} p_{o, i}$ in the continuum dynamics.

It is interesting to note that the fluctuation of $X$ (Fig. $5(\mathrm{a}))$ and $\tilde{G}_{o}$ (Fig. 5 (b)) when $k=6 \mathrm{pN} / \mathrm{nm}$ is lower than the value for the non-coupling case $k=0$. They fluctuate much less if the system is in the amplitude death state $(k=6 \mathrm{pN} / \mathrm{nm})$ compared to the noncoupling $(k=0)$ or collectively oscillating state $(k=2 \mathrm{pN} / \mathrm{nm})$. With the periodic stimulus $F_{\text {ext }} \neq 0$, the response of the amplitude death state $(k=6 \mathrm{pN} / \mathrm{nm})$ is much stronger than the uncoupled case $(k=0)$, while it is slightly weaker than the collectively oscillating case $(k=2 \mathrm{pN} / \mathrm{nm})$.

The signal amplification and noise reduction which is shown in Fig. 5 can be quantified by the signal-to-noise ratio (SNR). We calculate the power spectra of the mechanical displacement of the hair bundles,

$$
S_{X}(f)=\frac{1}{N} \sum_{i}^{N}\left|X_{i}(f)\right|^{2},\left|X_{i}(f)\right|=\frac{1}{T_{a}} \int_{0}^{T_{a}} x_{i}(t) e^{i 2 \pi f t} d t
$$


where $T_{a}$ is the time period for the Fourier transformation. $i$ is the index of a hair bundle. We plot $S_{X}(f)$ for a pure tone signal $(f=12 \mathrm{~Hz})$ of amplitude $0.2 \mathrm{pN}$. When a weak signal is applied to the uncoupled and weakly coupled bundles, the signal is buried in the noisy fluctuations as shown in Fig. $6((\mathrm{a}),(\mathrm{b}))$. But, if the acoustic signal is applied to stronglycoupled hair bundles(Fig. 6 (c)), the signal gets clearly exposed due to the reduction of the noisy fluctuations. SNR is defined by

$$
S N R=\lim _{\Delta f \rightarrow 0} \frac{S_{X}(f)}{\frac{1}{\Delta f} \int_{f-\Delta f / 2}^{f+\Delta f / 2} S_{X}\left(f^{\prime}\right) d f^{\prime}},
$$

where $f$ is the frequency of the external stimulus. $F_{\text {ext }}(t)=F \sin (2 \pi f t)$ is added on the right hand side of Eq.(1). We obtained the power spectra averaged over 50 different trials.

In Fig. 6 (d), we show that the SNR tends to increase with the coupling strength $k$. Due to the amplitude death, the increase of the SNR is enhanced. For the case of $N_{c h}=20$, $\gamma=10 \mathrm{~ms}^{-1}$, which has been used so far, one can see this enhancement of the SNR as the coupling strength $k$ increases. The increases of SNR is more enhanced as $k$ goes beyond 4 $\mathrm{pN} / \mathrm{nm}$, which enters the amplitude death region.

Fig. 6 (d) (and Supplemental Material Fig. 7) shows that the channel gating noise becomes weaker as the channel relaxation rate $\gamma$ increases and the number of channels per bundle $N_{c h}$ decreases. Thus, the SNR for $\gamma=200 \mathrm{~ms}^{-1}$ with single channel $N_{c h}=1$ looks similar for the bundles with 20 channels with $\gamma=10 \mathrm{~ms}^{-1}$. Thus, if the hair bundle has only one channel with the usual relaxation rate $\gamma=10 \mathrm{~ms}^{-1}$, then we do not see the amplitude death phenomenon due to the strong noise. In this case, the enhancement of the SNR is not significant as shown in Fig. $6(d)$.

\section{THERMAL NOISE vs TRANSDUCTION CHANNEL GATING NOISE}

The sources of the noise can be divided into those which arise from thermal fluctuation associated with the Brownian motion of hair bundles, and fluctuation associated with the stochastic nature of channel gating. Fluctuation associated with the stochastic activity of molecular motors also exists but its strength is relatively weak compared to the thermal noise [5], which we do not discuss here. While the thermal noise was considered in an earlier work [1], we have investigated the role of the stochastic channel noise so far in this work. Now, let us compare the two different noise sources, by including the thermal noise force[1]. 
This can be done by replacing Eq.(1) with

$$
\begin{aligned}
m \ddot{x}_{i} & =-m \gamma_{m} \dot{x}_{i}+k\left(x_{i+1}-2 x_{i}+x_{i-1}\right)+f_{\mathrm{HB}, i} \\
& +f_{N, i}(t)
\end{aligned}
$$

$f_{N, i}(t)$ is the thermal noise force which is exerted on the $i$-th hair bundle and satisfies the equipartition theorem. The noise force has the finite correlation time $\tau_{c}$ and it has the correlation function in the form of

$$
<\overline{f_{N, i}(t) f_{N, j}(t+\tau)}>=\frac{2 k_{B} T \tilde{\lambda} \delta_{i, j}}{e^{\left(\frac{\tilde{\lambda} \tau_{c}}{2 m}\right)^{2}} \operatorname{erfc}\left(\frac{\tilde{\lambda} \tau_{c}}{2 m}\right)} \frac{1}{\sqrt{\pi} \tau_{c}} e^{-\left(\tau / \tau_{c}\right)^{2}}
$$

where $\tilde{\lambda}=\lambda+m \gamma_{m}$ and $\operatorname{erfc}(\mathrm{x})$ is the complementary error function. (see Supplemental Material of Ref.[1]) $\tau_{c}$ is the correlation time of the thermal noise force which acts on the mechanical motion of the hair bundle. This value in many cases is assumed to be zero for the simplicity of the calculation. In reality, $\tau_{c}$ depends on the detail of the environment of the hair bundle. In this work, we choose a value close to a channel relaxation rate $\gamma^{-1}$.

Fig. 7 (a) shows that the channel gating noise can be more or less important to the mechanical fluctuations $\sigma_{X}$ compared to the thermal noise depending on the number of channels per bundle. Our numerical estimation for $N_{c h}=20$ shows that the mechanical noise power $S_{X}(f)$ due to the channel gating is weaker than the noise due to the thermal noise force (see Supplemental Material Fig. 4, 5, and 6). However, this situation is opposite when we consider the fluctuation of the number of open channels $\sigma_{G}$, which is important to the transduction ion current in a neuronal signal. $\sigma_{G}$ is defined by

$$
\begin{aligned}
\sigma_{G}^{2} & =\frac{1}{N} \sum_{i} \sigma_{G, i}^{2} \\
\sigma_{G, i}^{2} & =<\left(G_{i}-<G_{i}>_{t}\right)^{2}>_{t} .
\end{aligned}
$$

We find that the channel gating noise is the more important noise source to the number of open channels compared to the thermal Brownian noise force (Fig. 7 (b)). The channel gating noise contribution to $\sigma_{G}$ becomes weakened as $N_{c h}$ increases. Thus, for the case of $N_{c h}=20$, we see that the thermal noise force and the channel gating noise are in comparable strength. When the two noise sources coexist, the fluctuation of hair bundles is given by the sum of the contribution of each source (see Supplemental Material Fig. 7). 


\section{CONCLUSION}

We developed a stochastic model for the dynamics of the coupled hair bundles with channel gating noise. As in the continuum model for hair bundle dynamics[1], there exists a transition from the collective oscillation state to the amplitude death state above a certain coupling strength. The transition is also evidenced by the fact that the velocity distribution change from the half-Lorentzian distribution to the Gaussian distribution. The relative importance of the stochastic channel gating noise is compared to the thermal noise force on the hair bundle displacement and the number of open channels. We find that the stochastic channel noise can be reduced by increasing the number of the ion channels per hair bundle. For the hair bundles which have a few tens of the ion channels, we conclude that the stochastic channel gating noise is sufficiently weak so that the amplitude death phenomenon survives. Therefore, the enhancement of the signal-to-noise ratio of coupled hair bundles would be possible through the amplitude death phenomenon even with the stochastic channel noise.

\section{Acknowledgments}

This work was supported by the National Research Foundation of Korea (NRF) grant funded by the Korea government(MEST) (No.2011-0009557). We thank Jung-Wan Ryu, Jan Wiersig, and Joel Rasmussen for helpful discussion and careful reading of our manuscript.

[1] K.-H. Ahn, J. R. Soc. Interface, 10, 20130525 (2013).

[2] R. Fettiplace and C. M. Hackney, Nat. Neurosci., 7, 19 (2006).

[3] D. Maoiléidigh and F. Jülicher, J. Acoust. Soc. Am., 128, 1175 (2010).

[4] P. Martin, Active Hair-Bundle Motility of the Hair Cells of Vestibular and Auditory Organs, Springer Handbook of Auditory Research, 30, 93 (2007).

[5] B. Nadrowski, P. Martin, and F. Jülicher, Proc. Natl. Acad. Sci. U.S.A. 101, 12195 (2004).

[6] V. M. Eguíluz, M. Ospeck, Y. Choe, A. J. Hudpeth, and M. O. Magnasco, Phys. Rev. Lett. 84, $5232(2000)$.

[7] K. Dierkes, B. Lindner, and F. Jülicher, Proc. Natl. Acad. Sci. USA 10518669 (2008). 
[8] K. Dierkes, F. Jülicher, and B. Lindner, Eur. Phys. J. E, 35, 37 (2012).

[9] R. E. Mirollo and S. H. Strogatz, J. Stat. Phy. 60, 245 (1990).

[10] I. Ozden, S. Venkataramani, M. A. Long, B. W. Connors, and A. V. Nurmikko, Phys. Rev. Lett. 93, 158102 (2004).

[11] V. Resmi, G. Ambika, and R. E. Amritkar, Phys. Rev. E 84, 046212 (2011).

[12] A. N. Pisarchik, Phys. Lett. A 318, 65 (2003).

[13] Y. Song, J. Xu and T. Zhang, CHAOS 21, 023111 (2011).

[14] J.-W. Ryu, D.-S. Lee, Y.-J. Park, and C.-M. Kim, J. Kor. Phys. Soc., 55, 395 (2009).

[15] M. D. Wei and J. C. Lun, Appl. Phys. Lett. 91, 061121 (2007).

[16] Z. Neufeld, I. Z. Kiss, C. Zhou and J. Kurths, Phys. Rev. Lett. 91, 084101 (2003).

[17] R. Herrero, M. Figueras, J. Rius, F. Pi and G. Orriols, Phys. Rev. Lett. 84, 5312 (2000).

[18] Z. Hou and H. Xin, Phys. Rev. E 68, 055103(R) (2003).

[19] R. Karnatak, R. Ramaswany and A. Prasad, Phys. Rev. E 76, 035201 (2007).

[20] A. Prasad, M. Dhamala, B. M. Adhikari, R. Ramaswamy, Phys. Rev. E 81, 027201 (2010).

[21] A. J. Ricci, A. C. Crawford, and R. Fettiplace, Neuron, 40, 983 (2003).

[22] D. Clausznitzer, B. Lindner, F. Julicher, and P. Martin, Phys. Rev. E, 77, 041901 (2008).

[23] M. S. Smotherman, and P. M. Narins, J. Experi. Bio. 203, 2237 (2000).

[24] P. S. Steyger, P. G. Gillespie, and R. A. Baird, J. Neurosci., 15, 4603 (1998).

[25] A. J. Hudspeth, Sensory Transduction, (Rockefeller Univ. Press, New York), 357-370 (1992).

[26] P. Martin, A. D. Mehta, A. J. Hudspeth, Proc. Natl Acad. Sci. USA 97, 12026 (2000).

[27] N. Hacohen, J. A. Assad, W. J. Smith. D. P. Corey, J. Neurosci., 9, 3988, (1989). 

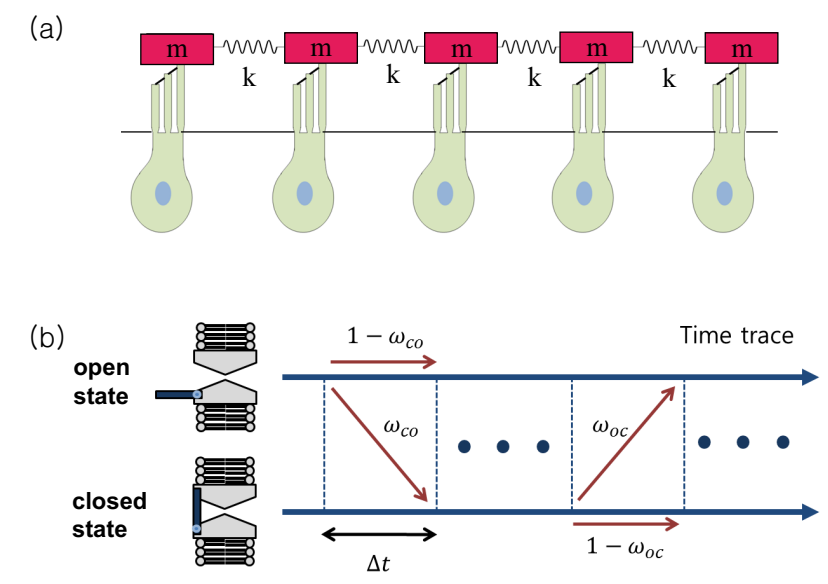

FIG. 1: (a) A model for coupled hair bundles. (b) Schematic drawing for Markov analysis of stochastic simulation for the coupled hair bundles dynamics. 

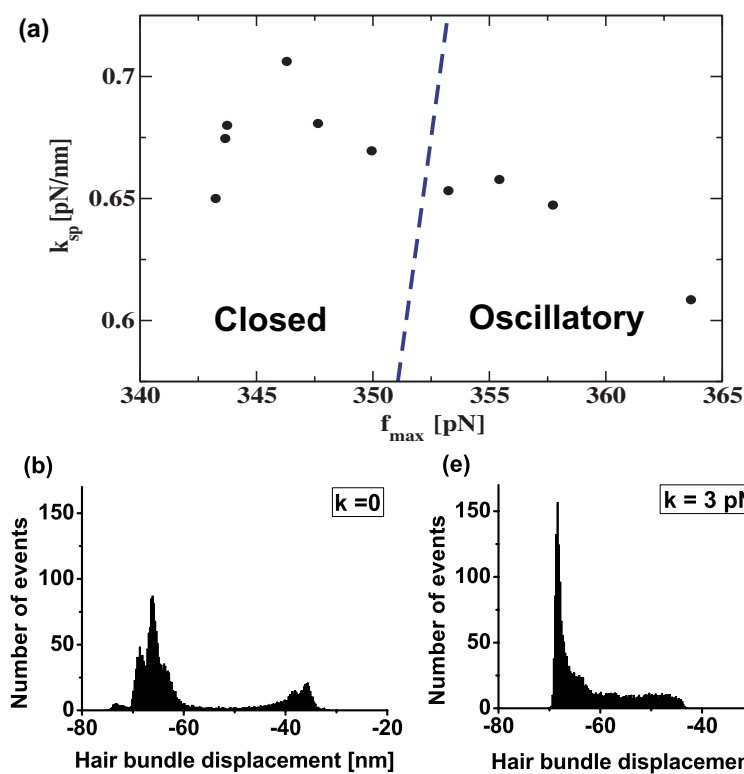

(e)
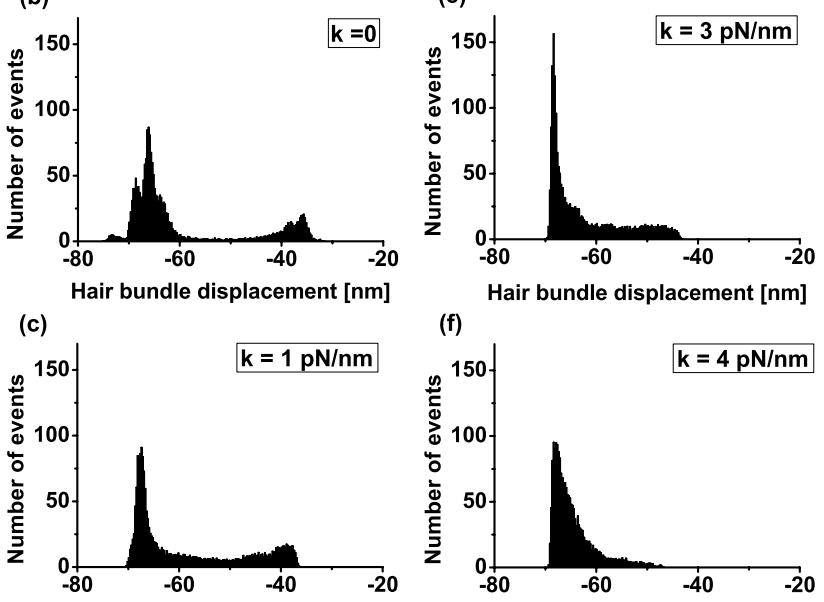

(f)
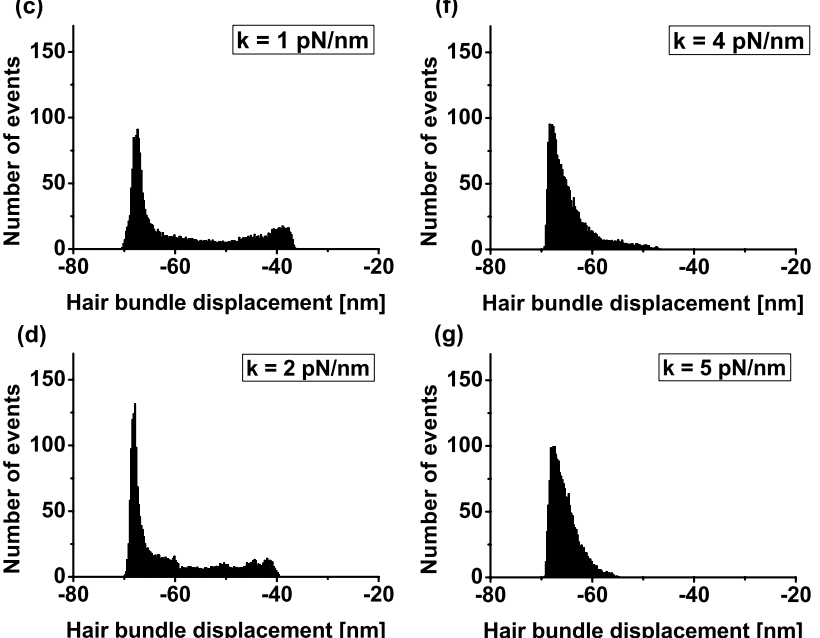

FIG. 2: (a) The parameter distribution for the pivotal stiffness of hair bundles $k_{s p}$ and the maximum forces $f_{\max }$ of molecular motors. We use this distribution for all simulations in this paper. $(\mathrm{b}) \sim(\mathrm{g})$ The histograms show the number of events which are counted every 1 millisecond for 2 seconds for (b) incoherent $(k=0),(\mathrm{c}) \sim(\mathrm{f})$ locking (or collectively oscillating) $(k=1 \mathrm{pN} / \mathrm{nm}, k=2 \mathrm{pN} / \mathrm{nm}$, $k=3 \mathrm{pN} / \mathrm{nm}$, and $k=4 \mathrm{pN} / \mathrm{nm})$, and (g) amplitude death region ( $k=5 \mathrm{pN} / \mathrm{nm}$ ) of 10 hair bundles. 


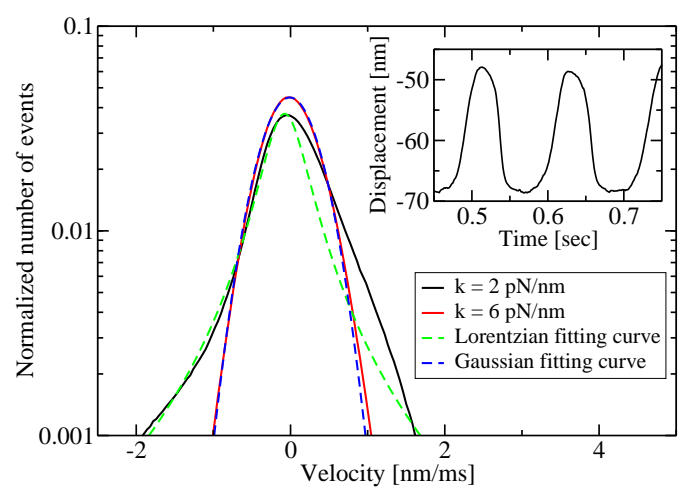

FIG. 3: The velocity distribution for amplitude death $(k=6 \mathrm{pN} / \mathrm{nm})$, locking $(k=2 \mathrm{pN} / \mathrm{nm})$ of 10 coupled hair bundles. The green dashed line is a fitting curve for the velocity distribution for locking oscillation(the black solid line) which is a half-Lorentzian curve. The blue dashed line is a Gaussian fitting curve for the velocity distribution for the amplitude death state (the red solid line). [inset] The averaged displacement of the coupled hair bundles at $k=2 \mathrm{pN} / \mathrm{nm}$ as a function of time.

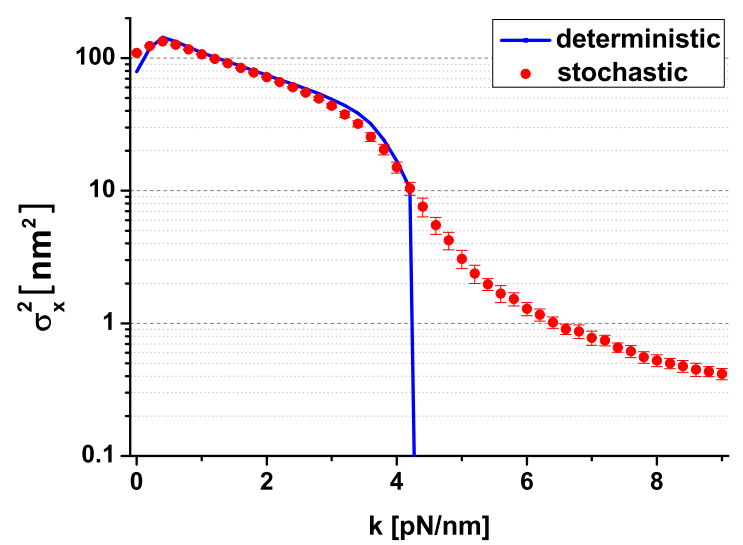

FIG. 4: The averaged variance for the displacement of the coupled hair bundles as a function of the inter-band coupling strength $k$. 
(a)

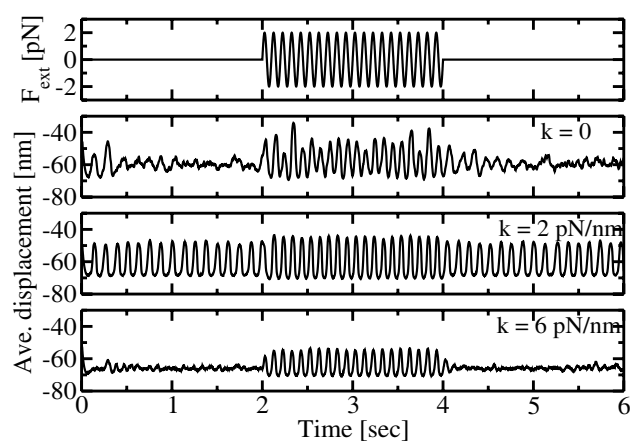

(b)

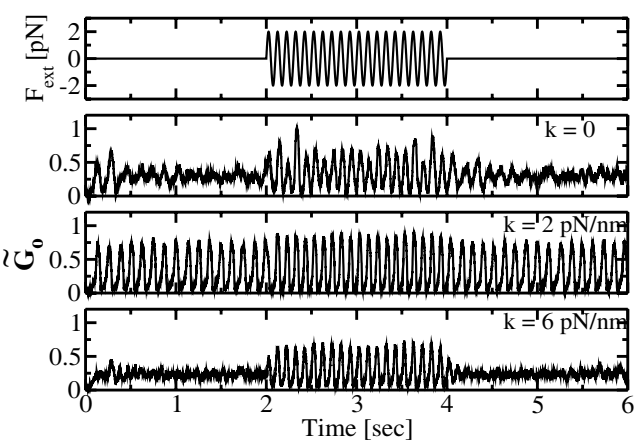

FIG. 5: (a) The average displacements $X=\frac{1}{N} \sum_{i} X_{i}$ for amplitude death $(k=6 \mathrm{pN} / \mathrm{nm})$, collectively oscillating $(k=2 \mathrm{pN} / \mathrm{nm})$, and uncoupled $(k=0)$ states of 10 coupled hair bundles. The top of the figure is the external force acting on the hair bundles. (b) The open channel ratio $\tilde{G}_{o}$ of 10 coupled hair bundles. 

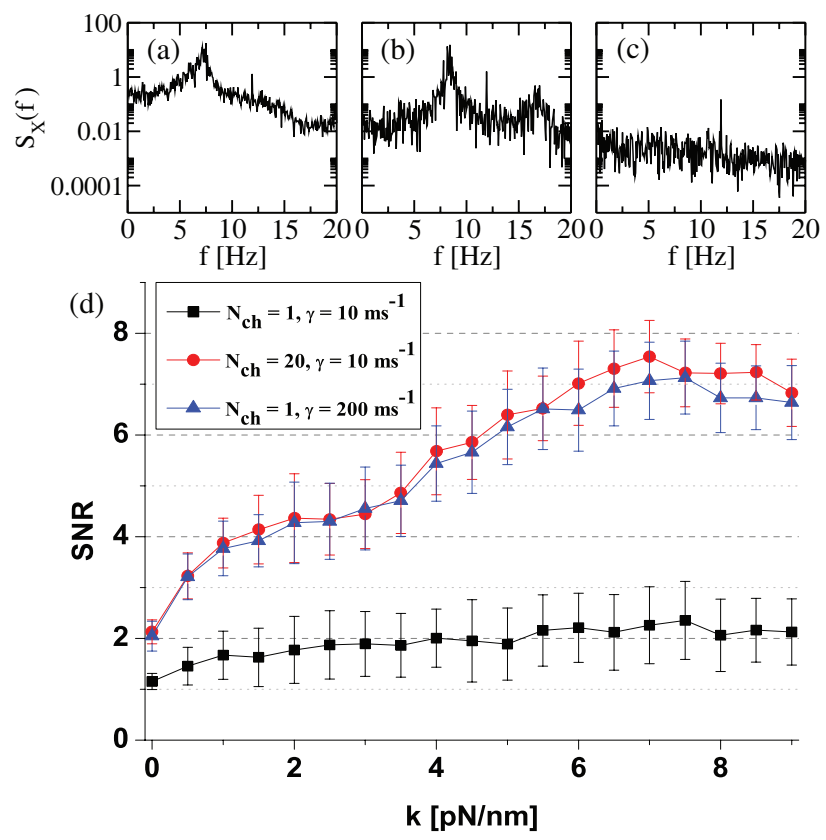

FIG. 6: (a-c) The power spectra of mechanical displacement $S_{X}(f)$ as a function of frequency $f$ when an external force is applied. The coupling strengths are (a)k=0, (b) $k=3 \mathrm{pN} / \mathrm{nm},(\mathrm{c}) k=9$ $\mathrm{pN} / \mathrm{nm}$. (d) SNR as a function of the coupling strength $k$. We used a $0.2 \mathrm{pN}$ external force with frequency of $12 \mathrm{~Hz}$ in our simulations (a-e). We obtained the power spectra averaged over 50 different trials. 

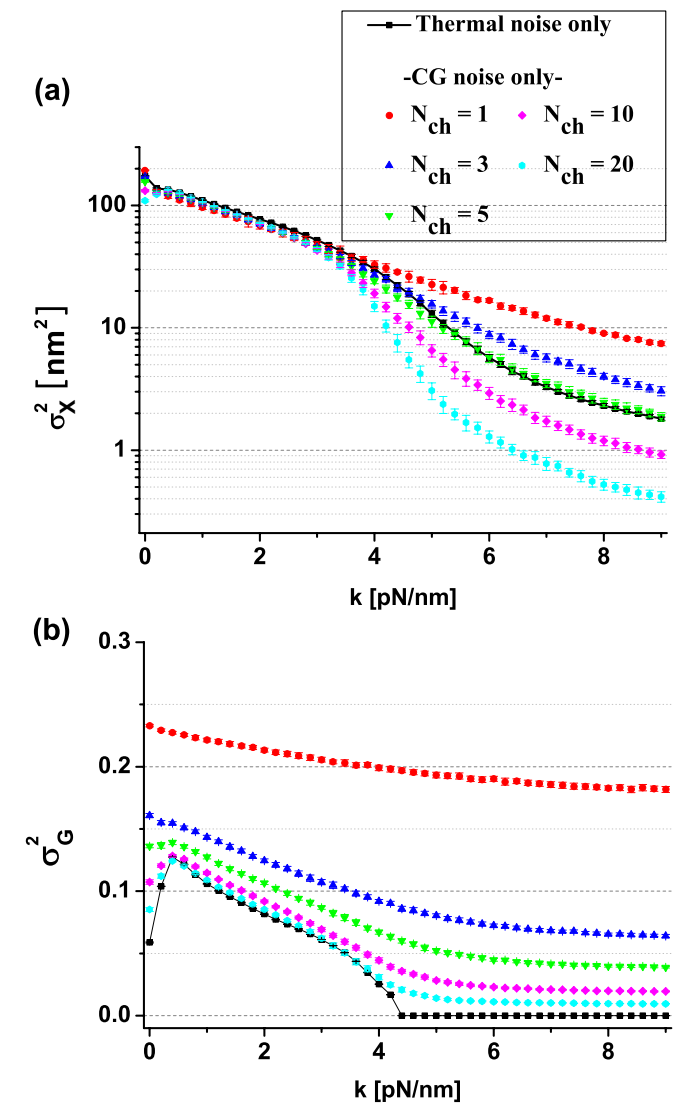

FIG. 7: (a) The variance $\sigma_{x}$ of the hair bundles' displacement as a function of the coupling strength $k$ (b) The variance $\sigma_{G}$ of the number of the open transduction channels as a function of the coupling strength. The black square dots are for the case when only the thermal noise exists. When only channel gating noise exists we plot the variances for the various number of channels. The error bars are obtained by 50 different trials. We used a correlation time $\tau_{c}=0.14 \mathrm{~ms}$ for the thermal noise and a relaxation rate $\gamma=10 \mathrm{~ms}^{-1}$ for the stochastic channel gating noise. In both case, as the number of ion channels per hair bundle increases, the variance decreases. 


\begin{tabular}{|c|c|c|c|}
\hline parameter & definition & value & Ref. \\
\hline$m$ & mass of one unit of cross-cut membrane & $2 \mu \mathrm{g}$ & \\
\hline$\gamma_{m}$ & friction constant per mass of the membrane & $0.5 \mathrm{~ms}^{-1}$ & \\
\hline$k$ & inter-bundle coupling strength & $0-9 \mathrm{pN} \mathrm{nm}^{-1}$ & \\
\hline$N$ & size of the one-dimensional hair bundle array & 10 & \\
\hline$N_{c h}$ & The number of channels per hair bundle & 20 & \\
\hline$\lambda$ & friction of a hair bundle & $2.8 \mu \mathrm{N} \mathrm{s} \mathrm{m}^{-1}$ & [5] \\
\hline$\lambda_{a}$ & friction of adaptation motors & $10 \mu \mathrm{N} \mathrm{s} \mathrm{m}{ }^{-1}$ & {$[27]$} \\
\hline$k_{g s}$ & combined gating spring stiffness & $0.75 \mathrm{pN} \mathrm{nm}^{-1}$ & {$[26]$} \\
\hline$<k_{s p}>$ & mean value of hair bundle pivot stiffness & $0.65 \mathrm{pN} \mathrm{nm}^{-1}$ & {$[26]$} \\
\hline$\delta k_{s p}$ & variance of hair bundle pivot stiffness & $0.05 \mathrm{pN} \mathrm{nm}^{-1}$ & \\
\hline$<f_{\max }>$ & mean value of maximal motor force & $350 \mathrm{pN}$ & [5] \\
\hline$\delta f_{\max }$ & variance of maximal motor force & $7.14 \mathrm{pN}$ & \\
\hline$D$ & gating spring elongation & $60.9 \mathrm{~nm}$ & {$[26]$} \\
\hline$S$ & strength of the calcium feedback & 0.65 & [5] \\
\hline$T$ & temperature & $300 \mathrm{~K}$ & \\
\hline$A$ & $\begin{array}{l}\text { constant associated with the intrinsic free energy dif- } \\
\text { ference }\end{array}$ & $\exp (16.7)$ & {$[\underline{5}, 25$} \\
\hline$\delta$ & typical length associated with the channel gating & $4.53 \mathrm{~nm}$ & {$[\underline{5}, 25$} \\
\hline$\gamma$ & channel relaxation rate & $10 \mathrm{~ms}^{-1}$ & \\
\hline$\Delta t$ & time interval for stochastic simulation & $0.04 \mathrm{~ms}$ & \\
\hline
\end{tabular}

TABLE I: List of the parameter values for the simulations 